\title{
Produção científica em avaliação psicológica no contexto escolar
}

\author{
Avaliação psicológica no contexto escolar
}

\author{
Katya Luciane de Oliveira \\ Acácia Aparecida Angeli dos Santos \\ Ana Paula Porto Noronha \\ Evely Boruchovitch \\ Cláudia Araújo da Cunha \\ Marucia Patta Bardagi \\ Simone F. da Silva Domingues
}

\begin{abstract}
Resumo
Esta pesquisa objetivou analisar a produção científica em avaliação psicológica no contexto escolar publicada em 234 artigos de sete periódicos científicos indexados. A análise baseou-se em alguns critérios da metaciência, a saber, autoria, temática, discurso e análise dos tipos de avaliações. Os resultados evidenciaram que em alguns periódicos há maior concentração de publicações sobre a temática, acentuada nos últimos anos. A participação feminina foi predominante na autoria dos artigos e detectou-se ampla diversificação nos propósitos e contextos nos quais os testes psicológicos têm sido usados. Quanto ao tipo de avaliação, os instrumentos psicométricos foram os mais utilizados, sendo freqüente, também, o emprego de entrevistas e observação. A técnica projetiva foi empregada em apenas $2,3 \%$ das investigações. Sugere-se que outros estudos similares sejam realizados, visando a monitorar a expansão da área de avaliação psicológica no Brasil.

Palavras-chave: avaliação psicológica; medida, ambiente educacional.
\end{abstract}

\section{Scientific production in psychological evaluation in the school context}

\begin{abstract}
This paper analyses the scientific production in psychological evaluation in the school context published in 234 articles from seven scientific indexed periodicals. The analysis was carried out according to some metascientific criteria such as: authorship, thematic, discourse, and exam of the different types of evaluation, to name a few. Results evinced not only a greater concentration of publications regarding to educational psychology evaluation in certain journals, but also an increase of these types of studies recently as well. It was also found that most of the authors were females and that psychological tests were used both in a variety of contexts and with diverse purposes. Psychometric instruments were the most frequently employed. Interviews and observations were also used. Projective techniques were reported only in $2.3 \%$ of the investigations. It is recommended that similar studies be conducted in order to better monitor the expanding area of psychological evaluation in Brazil.
\end{abstract}

Keywords: psychological assessment; measurement; school environment.

\section{Producción científica en evaluación psicológica en el contexto escolar}

\section{Resumen}

Esta investigación tuvo como objetivo analizar la producción científica en evaluación psicológica en el contexto escolar publicada en 234 artículos de siete revistas indexadas. El análisis se basó en algunos criterios de la meta-ciencia, son ellos, autor, temática, discurso y análisis de los tipos de evaluaciones. Los resultados mostraron que en algunas revistas hay una concentración mayor de publicaciones sobre la temática que se intensificaron en los últimos años. La participación femenina fue predominante en los autores de los artículos y se detectó una gran diversificación en los propósitos y contextos en los cuales los testes psicológicos han sido utilizados. En relación al tipo de evaluación, los instrumentos psicométricos fueron los más utilizados, siendo también frecuente la utilización de entrevistas y observaciones. La técnica proyectiva fue usada en apenas $2,3 \%$ de las investigaciones. Se sugiere que sean realizados otros estudios semejantes, con el objetivo de monitorear la expansión del área de evaluación psicológica en el Brasil.

Palabras clave: evaluación psicológica; medida; ambiente educacional. 


\section{Introdução}

Monitorar a produção científica em âmbito nacional e internacional é muito importante para avaliar o crescimento das diversas áreas do conhecimento. Uma das formas de se obter uma medida real desse crescimento é pela verificação da quantidade de artigos publicados em periódicos científicos indexados. A veiculação da produção científica depende de políticas de gestão científica, definidoras do papel das agências de fomento quanto ao desenvolvimento, à finalização e à publicação das pesquisas realizadas no país (Oliveira Filho, Hochman, Nahas \& Ferreira, 2005; Witter, 2005; Yamamoto, Souza \& Yamamoto, 1999).

Vale, entretanto, ressaltar que dentre as principais agências destacam-se a Coordenação de Aperfeiçoamento de Pessoal de Nível Superior (CAPEs), o Conselho Nacional de Desenvolvimento Científico e Tecnológico (CNPq) e a Fundação de Amparo à Pesquisa do Estado de São Paulo (FAPESP). Freitas (1998) observa que a região onde há maior concentração de produções científicas é a Sudeste. Essa região agrega $54 \%$ dos profissionais envolvidos com pesquisas, sendo que no cenário atual essa é a região que ainda concentra a maior parte dos financiamentos e o maior número de veículos de comunicação científica.

Vários estudos têm procurado analisar a produção científica em diversas áreas do conhecimento (Bariani, Buin, Barros \& Escher, 2004; Coimbra Jr., 1999; Figueira, Leta \& De Meis, 1999; Freitas, 1998; Meneghini, 1998; Meneghini \& Fonseca, 1990; Neves, Almeida, Chaperman \& Batista, 2002; Noronha, 1998; Noronha, Vendramini \& Freitas, 2004; Sampaio, Sabadini \& Linguanotto, 2002; Santos, Oliveira \& Joly, 2003; Zanella \& Titon, 2005, entre outros). Contudo, a indisponibilidade de acesso a diversas bases de dados nacionais compromete a acuidade da medida no cenário científico.

Atualmente o SciELO é um importantíssimo meio eletrônico da divulgação da produção nacional, recurso bastante utilizado por agregar parte significativa da produção científica brasileira. Outras bases que se destacam no panorama da divulgação da ciência são o Psyclnfo, atrelado à American Psychological Association e a base de dados LILACS e MEDLINE vinculada à National Library of Medicine e a LILACS que é por ela produzida. Outra alternativa importante é oferecida pelo Institute for Scientific Informaticon (ISI), em cuja base são inseridos periódicos de diversas áreas do conhecimento, após uma seleção rigorosa da qualidade de cada um. Nos últimos anos têm havido grande preocupação dos cientistas brasileiros em dar visibilidade às suas publicações, sendo a escolha de revistas indexadas no $|S|$ um critério decisivo para a submissão dos manuscritos (Izique, 2002; Witter, 2005, 2006).

No entanto, desde a criação da Biblioteca Virtual em Psicologia (BVS-PSI), em 200I, houve um diferencial no que se refere à divulgação da produção de conhecimento científico na psicologia, que tornou a área distinta das demais no que se refere à divulgação da produção científica nacional. $O$ apoio tecnológico do Centro Latino Americano de Informação em Ciências da Saúde (BIREME) foi fundamental para que a BVS-PSI fosse implementada (Costa, 2006). Posteriormente foi criada a base de dados PePSIC, resultante de iniciativa da própria BVS-PSI em pareceria com a Associação Brasileira de Periódicos Científicos em Psicologia (ABECIP), estruturada de acordo com a metodologia SciELO, que permitiu o acesso livre a textos completos de 39 títulos de revistas da psicologia, possibilitando a utilização de mecanismos de metabusca para recuperação dos textos pelos descritores, títulos e/ou autores. Esses recursos permitiram o desenvolvimento de pesquisas que levantassem mais precisamente a produção científica.

A bibliometria mensura a ciência por meio da abordagem empírico-analítica da produção científica, de tal sorte que a cumulatividade do conhecimento, sua divulgação e impacto são os focos dessa análise (Figueira e cols., 1999; Mostafá \& Máximo, 2003). No que tange ao conteúdo da publicação, a metaciência, segundo Witter (1999), possibilita analisar as dimensões da produção científica em termos da qualidade do conteúdo dessa publicação, permitindo a visualização de mudanças no fluxo da produção em subáreas do conhecimento e, até mesmo, de temas específicos.

Estudos têm constatado que as pesquisas em psicologia escolar e educacional têm se proposto a ex- 
plorar as múltiplas dimensões dos aspectos cognitivos, afetivos, culturais e sociais relacionados. A investigação dessas variáveis tem fortalecido a compreensão das diversas esferas implicadas no escopo do processo escolar e educacional e, freqüentemente, são utilizados instrumentos de avaliação psicológica para acessar direta e/ou indiretamente os fenômenos abordados (Joly, 2000). É importante ressaltar que essa subárea tem ampliado o seu espaço na produção científica nacional (Azevedo \& Aguiar, 200I; Bariani e cols., 2004; Neves e cols., 2002; Oliveira, Cantalice, Joly \& Santos, 2006; Santos e cols., 2003; Witter, 1979; Witter, 1996).

Nenhuma atenção, no entanto, tem sido dada ao rastreamento da produção em avaliação psicológica no contexto escolar e educacional. Esse fato talvez possa ser mais bem compreendido, considerando-se que no passado recente o uso de testes era visto com muita desconfiança como instrumento auxiliar nas avaliações realizadas na situação escolar. Muitos psicólogos atuantes na área encaravam o teste psicológico como um recurso meramente classificatório. Sisto, Sbardelini e Primi (200I) refutam essa idéia quando destacam que o teste psicológico deve ser mais um recurso e não o único no qual o psicólogo vai utilizar em um processo de avaliação psicológica. Nesse sentido, o teste deveria ser mais um elemento que deveria ser agregado a outras formas qualitativas de coleta de informações, como entrevistas e observações, por exemplo.

Em estudo recente de meta-análise, realizado por Souza Filho, Belo e Gouveia (2006), foi analisada a utilização dos testes psicológicos na literatura científica brasileira nos últimos anos. A fonte utilizada foi a base de Periódicos CAPES, disponível em fevereiro de 2005, abrangendo os artigos publicados entre os anos de 2000 e 2004. Os resultados demonstraram predominância de trabalhos que não utilizaram nenhum teste. Dentre os que relatam o uso de testes, houve um equilíbrio entre aqueles que os utilizam de forma direta e indireta. Os autores destacaram que a maior concentração dessas produções está situada na Região Sudeste e que entre as universidades mais produtivas estão instituições públicas e privadas, especialmente aquelas que têm um histórico pautado pelo interesse na área da avaliação psicológica. Conclui-se, de forma geral, que a utilização dos testes psicológicos no contexto da produção nacional ainda é modesta e está, em grande parte, restrita aos âmbitos acadêmicos mais intensamente dedicados ao estudo dos testes.

No ano de 200I, houve uma série de eventos que podem ser considerados marcantes para a área de avaliação psicológica. Assim, concomitantemente, foi promulgada a primeira resolução do Conselho Federal de Psicologia (CFP) sobre a construção e o uso de testes psicológicos, criado o Instituto Brasileiro de Avaliação Psicológica (IBAP) e aprovado pela CAPES, o primeiro curso de mestrado em psicologia, com área de concentração em avaliação psicológica.

A base de informação, analisada por Souza Filho e cols. (2006) sobre o uso de testes psicológicos na produção científica em psicologia no Brasil, incluiu relatos de pesquisas realizadas em anos anteriores à data de sua publicação. É importante lembrar que a publicação de um artigo é resultante da discussão de dados que são coletados num determinado momento e depois organizados dentro dos parâmetros exigidos para esse suporte de informação. Além disso, há o cumprimento de uma série de passos em uma tramitação editorial complexa, que envolve desde a submissão do manuscrito aos pares que emitem seus pareceres às cegas até a própria editoração, que inclui a diagramação, revisão, nova conferência pelos autores, entre outros.

Sob essa perspectiva, observa-se que as informações avaliadas por Souza Filho e cols. (2006) refere-se a dados que, pelo menos parcialmente, devem ter sido coletados em época que antecedeu as mudanças mencionadas como marcantes para a área de avaliação psicológica. Considerando-se que outros trabalhos de meta-análise, citados neste texto, referentes à psicologia escolar não tiveram como objetivo focalizar o uso de testes, julgou-se pertinente investigar se no contexto educacional, e em especial, nesse período de transição na área, as produções publicadas em periódicos nacionais sofreram alterações em relação ao uso de instrumentos de avaliação psicológica, portanto, esse foi o objetivo deste estudo. 


\section{Método}

\section{Fontes}

Sete periódicos científicos na área de psicologia foram analisados, cujo critério de escolha foi o fato do periódico apresentar um conceito de avaliação ' $A$ ' nacional', com toda a sua coleção disponível. As revistas analisadas foram Estudos de Psicologia da PUCCampinas, Estudos de Psicologia de Natal, Psicologia Escolar e Educacional, Psicologia: Reflexão e Crítica, Psicologia em Estudo, Psico-USF e Psicologia: Ciência e Profissão.

\section{Procedimento}

Nesses periódicos buscou-se analisar somente os resumos dos artigos que trabalharam com avaliação psicológica nos contextos escolar e educacional. Para tanto, estabeleceu-se um período de 10 anos (1995-2004) para a realização da análise. O total de artigos levantados foi de 1603. Todavia, apenas 234 tratavam especificamente de publicações relacionadas à avaliação psicológica no contexto escolar/educacional.

Os periódicos foram analisados respeitando alguns critérios estabelecidos nos estudos realizados por Witter (1999). Desse modo, os seguintes itens foram considerados, Autoria, identificou- se a natureza da autoria (individual ou múltipla), bem como, o gênero dos autores; Temática, analisou-se a quantidade e a distribuição por temas de avaliação; Discurso, avaliou-se as palavras contidas no título do trabalho e número e escolaridade dos participantes; Análise das Avaliações, realizou-se a classificação do tipo de instrumentos empregados nas avaliações, bem como elencou-se os instrumentos utilizados.

\section{Resultados}

Os dados foram organizados em planilha e submetidos à estatística descritiva conforme o objetivo deste estudo. Para avaliar o universo geral de publicações efetuou-se a contagem da quantidade de artigos publicados por volume, em cada periódico, nos últimos 10 anos. As Tabelas 1 e 2 apresentam os dados obtidos.

Observa-se um aumento da quantidade de volumes a partir de 1999, bem como da quantidade de publicações. Salienta-se que em 1995 apenas algumas revistas já publicavam seus artigos, apresentando inclusive volumes bem anteriores ao ano citado.

As revistas que apresentaram maior quantidade de artigos publicados foram Psicologia: Reflexão e

Tabela I. Distribuição geral da quantidade de volumes, total de artigos publicados e média de publicações em avaliação psicológica

\begin{tabular}{cccc}
\hline Ano & $\begin{array}{c}\text { Quantidade de } \\
\text { Volumes }\end{array}$ & Total Ano & $\begin{array}{c}M \text { de Publicação } \\
\text { Ano }\end{array}$ \\
\hline 1995 & 4 & 67 & 16,7 \\
1996 & 4 & 96 & 24,0 \\
1997 & 6 & 122 & 20,3 \\
1998 & 6 & 142 & 23,7 \\
1999 & 7 & 146 & 20,8 \\
2000 & 7 & 149 & 21,3 \\
2001 & 7 & 181 & 25,8 \\
2002 & 7 & 231 & 33,0 \\
2003 & 7 & 229 & 32,7 \\
2004 & 7 & 240 & 34,3 \\
\hline
\end{tabular}


Tabela 2. Distribuição da publicação sobre avaliação psicológica no contexto escolar por periódico científico

\begin{tabular}{|c|c|c|c|c|c|c|c|}
\hline Ano & $\begin{array}{l}\text { Estudos de } \\
\text { Psicologia } \\
\text {-PUC }\end{array}$ & $\begin{array}{l}\text { Estudos de } \\
\text { Psicologia } \\
\text { - Natal }\end{array}$ & $\begin{array}{l}\text { Psicologia } \\
\text { Escolar e } \\
\text { Educacional }\end{array}$ & $\begin{array}{c}\text { Psicologia: } \\
\text { Reflexão e } \\
\text { Crítica }\end{array}$ & $\begin{array}{c}\text { Psicologia } \\
\text { em Estudo } \\
\text { de } \\
\text { Maringá }\end{array}$ & $\begin{array}{c}\text { Psico- } \\
\text { USF }\end{array}$ & $\begin{array}{c}\text { Psicologia: } \\
\text { Teoria e } \\
\text { Pesquisa }\end{array}$ \\
\hline 1995 & 22 & - & - & 17 & - & - & 28 \\
\hline 1996 & 19 & - & 8 & 19 & 7 & 11 & 32 \\
\hline 1997 & 19 & 16 & 6 & 24 & 15 & 12 & 30 \\
\hline 1998 & 14 & 12 & 17 & 40 & 16 & 11 & 32 \\
\hline 1999 & 14 & 13 & 13 & 47 & 19 & 10 & 30 \\
\hline 2000 & 18 & 17 & 8 & 51 & 15 & 13 & 27 \\
\hline 2001 & 19 & 22 & 12 & 54 & 24 & 19 & 31 \\
\hline 2002 & 20 & 46 & 14 & 64 & 29 & 22 & 36 \\
\hline 2003 & 23 & 51 & 17 & 55 & 31 & 21 & 31 \\
\hline 2004 & 25 & 53 & 18 & 44 & 48 & 22 & 30 \\
\hline $\begin{array}{l}\text { Total de } \\
\text { artigos }\end{array}$ & 193 & 230 & 113 & 415 & 204 & 141 & 307 \\
\hline $\begin{array}{l}\text { Artigos que } \\
\text { envolveram } \\
\text { avaliação }\end{array}$ & 8 & 53 & 59 & 64 & 18 & 9 & 23 \\
\hline
\end{tabular}

Crítica e Psicologia: Teoria e Pesquisa. Vale lembrar que alguns periódicos não tiveram muitas publicações em razão de terem iniciado suas impressões mais tardiamente em relação àquelas que já apresentavam constância na publicação. No que tange à quantidade de artigos publicados na área de avaliação psicológica no contexto escolar e educacional, foram analisados 234. A revista Psicologia: Reflexão e Crítica também apresentaram mais artigos publicados $(27,3 \%)$ sobre o tema, sendo seguida pela Psicologia Escolar e Educacional $(25,2 \%)$ e pelos Estudos de Psicologia (22,6\%).

No que se refere à avaliação da autoria, entre os 234 artigos analisados, observou-se que $75,2 \%$ $(n=176)$ foram realizados com autoria múltipla e $24,8 \%(n=58)$ com autoria individual. Quanto ao gênero dos autores no total de trabalhos $(n=234)$, a maioria foi escrita por pessoas do sexo feminino $51,3 \%(n=120)$, seguida de publicações em parceria entre ambos os sexos $39,3 \%(n=39,3)$ e em menor percentual 9,4\% $(n=22)$ foram constatados artigos, cuja autoria foi apenas masculina. A análise pelo teste Qui-quadrado mostrou que a distribuição não era eqüitativa, considerando $\left[\chi^{2}(2,234)=65,33 ; p d \geq 0,00 I\right]$. Pelo resultado verificou-se que as mulheres apresentaram mais publicações na área do que os homens, mas ressalta-se que a parceria entre ambos também ocorre com freqüência.

A análise da Temática revelou a quantidade e a distribuição dos artigos, considerando os temas implicados na avaliação. A Tabela 3 mostra tal distribuição, salientando que alguns artigos trataram de duas ou mais temáticas ao mesmo tempo. Desse modo, todas as temáticas pesquisadas foram computadas totalizando 269 temas.

A análise do teste Qui-quadrado apontou que a distribuição das temáticas não era eqüitativa, tendo em vista $\left[\chi^{2}(\mid 2,269)=80,45 ; p d \geq 0,00 \mid\right]$. Nesse sentido, observou-se que a leitura e a escrita ainda são as 
Tabela 3. Distribuição da freqüência dos artigos considerando a temática pesquisada

\begin{tabular}{lcc}
\hline Categorias & F & $\%$ \\
\hline Leitura-escrita & 54 & 20,1 \\
Sucesso e fracasso escolar (diferentes facetas) & 33 & 12,3 \\
Aspectos cognitivos & 32 & 11,9 \\
Aspectos afetivo-emocionais & 30 & 11,1 \\
Habilidades sociais/interações comportamentais & 27 & 10 \\
Orientação vocacional, profissional, mercado de trabalho, interesses & 16 & 5,9 \\
profissionais & 15 & 5,6 \\
Sobre ensinar e aprender & 12 & 4,5 \\
Cidadania, ética, atitudes, valores, dilemas e regras morais & 12 & 4,5 \\
Questões do desenvolvimento & 9 & 3,3 \\
Criatividade & 7 & 2,6 \\
A avaliação e seus componentes & 6 & 2,2 \\
Motivação & 16 & 5,9 \\
Outros & & \\
\hline
\end{tabular}

temáticas mais investigadas. Ainda se manteve uma categoria Outros que reuniu as seguintes temáticas: conflito sóciocognitivo, educação ambiental, educação em saúde, expectativa de vida, faz de conta, histórias infantis, jogos recreativos/lúdicos, pesquisa ecológica, pesquisa intervenção e violência.

No que concerne ao Discurso foram avaliadas as palavras contidas nos títulos dos trabalhos, número e escolaridade dos participantes. Quanto às palavras do título, a análise dos 234 manuscritos evidenciou que um grande número de artigos $(64,1 \% ; n=150)$ ultrapassa o limite definido de doze vocábulos no título. No que diz respeito às demais categorias relativas ao Discurso, as Tabelas 4 e 5 mostram as análises realizadas. Vale esclarecer que no caso da análise da quantidade de participantes/sujeitos nas pesquisas e respectiva escolaridade, o número de artigos avaliados passou de 234 para 219. Essa diminuição ocorreu em função de que $15(6,4 \%)$ artigos não trabalharam com sujeitos de pesquisa. Essas publicações eram de cunho teórico ou documental, sendo que nesse caso, as pesquisas eram feitas em fontes documentais (disser- tações, teses, artigos, manuais de testes psicológicos, entre outros).

Observou-se que 21 trabalhos apresentaram, de forma genérica, os seus participantes, não especificando a quantidade de sujeitos incluídos no estudo. Foi também constatado que apenas 7 trabalhos foram realizados com amostras maiores que 1000 pessoas.

Ao verificar a escolaridade dos participantes a distribuição não foi novamente eqüitativa, tendo em vista $\left[\chi^{2}(8,219)=97,56 ; p d \geq 0,00\right.$ I $]$. Sob esse aspecto, evidenciou-se uma concentração na realização de estudos com participantes matriculados no ensino superior, seguido das primeiras séries do ensino fundamental ( $I^{\mathrm{a}}$ a $\left.4^{\mathrm{a}}\right)$. Todavia, estudos cujos participantes eram provenientes de diferentes fases de escolaridade, variando da pré-escola, ensino básico, médio e superior foram também encontrados.

A Tabela 6 mostra a Análise das Avaliações, na qual se realizou a classificação do recurso (tipo de instrumento) empregado nas avaliações. Cabe destacar que novamente o número de artigos focalizados sofreu uma mudança, passou de 234 para 222 na análise dessa 
Tabela 4. Análise do número de participantes/sujeitos nas pesquisas $(n=2 \mid 9)$

\begin{tabular}{lrc}
\hline Categorias do número de participantes & F & $\%$ \\
\hline De 1 a 10 & 19 & 8,7 \\
De 11 a 20 & 16 & 7,3 \\
De 21 a 30 & 9 & 4,1 \\
De 31 a 50 & 27 & 12,3 \\
De 51 a 100 & 39 & 17,8 \\
De 101 a 200 & 29 & 13,2 \\
De 201 a 300 & 14 & 6,4 \\
De 301 a 400 & 10 & 4,6 \\
De 401 a 500 & 8 & 3,6 \\
De 501 a 700 & 12 & 5,5 \\
De 701 a 1000 & 8 & 3,6 \\
Mais de 1000 & 7 & 3,3 \\
Indefinido & 21 & 9,6 \\
\hline
\end{tabular}

Tabela 5. Distribuição dos trabalhos em razão da etapa de escolarização dos participantes $(n=219)$

\begin{tabular}{lcc}
\hline Tipos de Avaliação & F & $\%$ \\
\hline Jardim da infância & 3 & 1,4 \\
Pré-escolar & 10 & 4,6 \\
Ensino fundamental (geral) & 26 & 11,9 \\
Ensino fundamental (de 1 a a $4^{\mathrm{a}}$ série) & 45 & 20,5 \\
Ensino fundamental (de $5^{\mathrm{a}}$ a $8^{\mathrm{a}}$ série) & 10 & 4,6 \\
Ensino médio & 12 & 5,5 \\
Ensino superior & 52 & 23,7 \\
Escolaridade mista & 37 & 16,9 \\
Escolaridade indefinida & 24 & 10,9 \\
\hline
\end{tabular}

modalidade. Esse dado é justificável, tendo em vista que alguns artigos documentais trabalharam com a análise de instrumentos de medida, à luz dos seus manuais. Nesse sentido, se o universo de 234 passou para 219 no caso da análise dos artigos que trabalharam com sujeitos de pesquisa, na análise do recurso empregado na avaliação, o montante se restringiu a $222(94,6 \%)$. Portanto, apenas três (I,2\%) eram puramente teóricos.

Recorreu-se mais uma vez ao Qui-quadrado para a análise da distribuição $\left[\chi^{2}(7,222)=243,57\right.$; $p d \geq 0,00 I$, verificando-se que houve diferença esta- 
Tabela 6. Análise dos artigos por recurso empregado na avaliação $(n=222)$

\begin{tabular}{lcc}
\hline Tipos de Avaliação & F & $\%$ \\
\hline Psicométrica/Fatorial & 90 & 40,5 \\
Questionários/Entrevista & 47 & 21,1 \\
Situações/problemas escolares & 42 & 18,9 \\
Utiliza vários recursos ao mesmo tempo & 28 & 12,7 \\
Observação & 5 & 2,3 \\
Situações/problemas propostos no computador & 5 & 2,3 \\
Projetiva & 3 & 1,3 \\
Roteiros & 2 & 0,9 \\
\hline
\end{tabular}

tisticamente significativa. Pela análise verificou-se que há uma tendência na utilização de instrumentos do tipo psicométrico/fatorial em detrimento de outros recursos de avaliação A lista completa de todos os instrumentos que foram utilizados nos artigos consta no Quadro I.

\section{Discussão e Conclusão}

Observou-se que a revista Psicologia: Reflexão e Crítica apresentou mais artigos publicados na área de avaliação psicológica no contexto escolar/educacional. Ao lado disso, a Psicologia Escolar e Educacional apareceu como segunda colocada nas publicações com esse foco e, em terceiro lugar a Estudos de Psicologia. Chama atenção o fato de que um periódico especializado em publicações na área escolar e educacional tenha apresentado segunda colocação no ranking de publicações dessa natureza. Contudo, esse fato pode ser entendido à luz da "idade" desses dois periódicos, e nesse sentido, parece plausível que a Psicologia: Reflexão e Crítica apresente um número superior. A Psicologia Escolar e Educacional teve seu primeiro volume publicado em 1996 e, a partir de então, passou por algumas estruturações, sendo que somente em 2000 começou a oferecer regularidade em suas publicações.

A análise da autoria indicou que as mulheres apresentaram uma maior prevalência nas publicações em relação aos homens, com confirmação estatisticamente significativa $(p d \geq 0,00 \mathrm{I})$. Esse resultado pode ser discutido sob a perspectiva da própria categoria profissional que abriga, em sua maioria, profissionais do gênero feminino. Desse modo, nada mais comum do que as mulheres terem apresentado mais trabalhos publicados do que os homens. Não se pode deixar de mencionar que a parceria entre ambos também ocorreu com freqüência nas publicações.

$\mathrm{Na}$ Temática se analisou a quantidade e a distribuição dos artigos, considerando os temas implicados na avaliação. Salienta-se que todos os temas tratados foram elencados nas categorias. $O$ tema mais pesquisado foi a avaliação da habilidade de leitura e escrita, embora outros tenham se mostrado como foco de interesse; dentre eles, a avaliação da interação e habilidade sociais, a avaliação das dificuldades de aprendizagem e também da formação profissional. Todavia, muitos temas ainda carecem de investigações, o que promoveria o crescimento deste tipo de recorte da avaliação aplicada à área escolar e educacional com a discussão e ampliação das questões envolvidas nesse contexto. Ressalta-se que é uma área que necessita de estudos sistemáticos de avaliação que forneçam subsídios para que os resultados obtidos por meio dela gerem práticas educativas mais eficazes.

Quanto às palavras contidas nos títulos dos trabaIhos, os resultados mostraram que a distribuição não foi eqüitativa. Desse modo, os títulos dos trabalhos 
Quadro I. Relação dos instrumentos utilizados na avaliação

\begin{tabular}{|c|c|c|}
\hline $\begin{array}{l}\text { Tipos de } \\
\text { Instrumentos }\end{array}$ & \multicolumn{2}{|c|}{ Instrumentos/Recursos } \\
\hline $\begin{array}{l}\text { Fatoriais/ } \\
\text { Psicométricos }\end{array}$ & 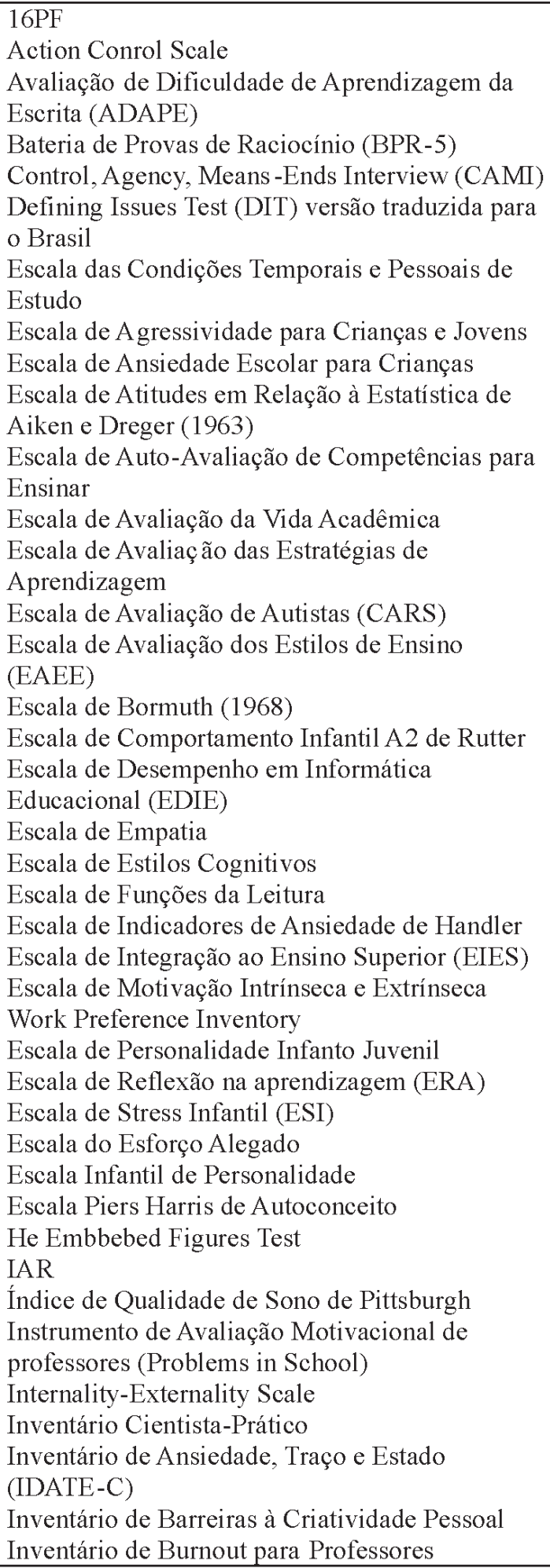 & $\begin{array}{l}\text { Revisado (CBP-R) } \\
\text { Inventário de Depressão Infantil (CDI) } \\
\text { Inventário de Habilidades Sociais (IHS) } \\
\text { Inventário de Incentivo à Criatividade } \\
\text { Inventário de Raciocínio Diagnóstico (IRD) } \\
\text { Inventário de Sintomas de Stress Infantil (ISSI) } \\
\text { Inventário de Traço de Ansiedade } \\
\text { Inventário de Vivências e Percepções de Estágio } \\
\text { (Versão para as Licenciaturas em Ensino) } \\
\text { Inventário Multimídia de Habilidade Sociais } \\
\text { para Crianças (IMHSC) } \\
\text { Inventário para Planejamento de Programas } \\
\text { Educacionais (PEP-R) } \\
\text { LIP } \\
\text { Maslach Burnout Inventory (MBI) } \\
\text { Medida de Atitudes Frente ao Consumo de } \\
\text { Materiais Pornográficos } \\
\text { Medida de Entusiasmo Frente à Leitura } \\
\text { Medida de Inteligência Emocional (MIE) } \\
\text { Medida sociométrica } \\
\text { Pavlovian Temperament Survey } \\
\text { Raven - Escala Especial } \\
\text { Raven - Escala Geral } \\
\text { Self-Directed Search (SDS) versão Brasileira } \\
\text { Son-R Test } \\
\text { STAXI } \\
\text { Teacher's Response Forma (TRF) } \\
\text { Teste Brasileiro de Provérbios (TBP-1989) } \\
\text { Teste da História incompleta } \\
\text { Teste de Cloze } \\
\text { Teste de Competência de leitura Silenciosa } \\
\text { (TeCoLeSi) } \\
\text { Teste de Competências em Leitura } \\
\text { Teste de Conceitos Básicos de Boehm } \\
\text { Teste de Memória Verbal } \\
\text { Teste de Prontidão para leitura } \\
\text { Teste de Raciocínio abstrato de Andriola e } \\
\text { Pasquali (1996) } \\
\text { Teste de Raciocínio verbal de Andriola e } \\
\text { Pasquali (1996) } \\
\text { Teste Diagnóstico de Habilidade do Pré-escolar } \\
\text { Teste do Desempenho Escolar (TDE) } \\
\text { Teste Metropolitano de Prontidão } \\
\text { Weste Sociométrico (brincar e fazer tarefas) } \\
\text { WISC III } \\
\text { WISC R } \\
\text { Young Self Report }\end{array}$ \\
\hline Projetivos & $\begin{array}{l}\text { Avaliação por Desenhos } \\
\text { Desenho da Casa-Árvore-Pessoa (HTTP) } \\
\text { Sistema Goodenough de Avaliação }\end{array}$ & $\begin{array}{l}\text { Teste das Pirâmides Coloridas de Pfister (TPC) } \\
\text { Teste Gestáltico Visomotor de Bender } \\
\text { Desenho da Figura Humana }\end{array}$ \\
\hline
\end{tabular}




\begin{tabular}{|c|c|c|}
\hline $\begin{array}{l}\text { Questionários/ } \\
\text { Entrevistas }\end{array}$ & $\begin{array}{l}\text { Anamnese médica } \\
\text { Cuestionario de Autocencepto Forma A (AFA) } \\
\text { Cuestionário de Depresion para Ninos (CDS) } \\
\text { Entrevistas Estruturadas } \\
\text { Entrevistas Semi-estruturadas } \\
\text { Questão traduzida e adaptada do Sef-Regulated } \\
\text { Learning Strictured Interview } \\
\text { Questionário de Atividade de Classe (CAQ) } \\
\text { Questionário de Estilo de Atribuição para } \\
\text { Crianças (CASQ) } \\
\text { Questionário de Informática Educacional (QIE) } \\
\text { em formato eletrônico } \\
\text { Questionário de Maturidade Vespertina } \\
\text { Questionário de Motivação no Ensino Superior } \\
\text { Questionário de Problemas de Comportamentos } \\
\text { de Achenbach (1991) }\end{array}$ & $\begin{array}{l}\text { Questionário de Processos de Aprendia gem } \\
\text { (QPA) } \\
\text { Questionário de Spini e Doise (1998) sobre } \\
\text { Envolvimento, Direitos Humanos e } \\
\text { Participações Sociais. } \\
\text { Questionário de Valores de Schwartz } \\
\text { Questionário de Valores Psicossociais } \\
\text { Questionário de Vivência Acadêmica (QVA) } \\
\text { Questionário do Comportamento de Ler } \\
\text { Questionário do Individulismo-Coletivismo } \\
\text { Questionário sobre lembranças e Histórias dos } \\
\text { Estudantes como leitores } \\
\text { Questionário sobre o Serviço de Atendimento ao } \\
\text { Universitário (SAL) }\end{array}$ \\
\hline & Roteiro de Avaliação da Atenção (RIA) & Roteiro de Observação \\
\hline Outros & $\begin{array}{l}\text { Adjetivos Auto-descritores } \\
\text { Avaliação do Repertório Básico para a } \\
\text { Alfabetização } \\
\text { Cartões de desenhos } \\
\text { Conjunto de Sentenças } \\
\text { Construção de Eqüidistâncias de Piaget } \\
\text { Desenho Livre com base em Figura Geométrica } \\
\text { Estilos de Pensar e Criar } \\
\text { Jogo das } 4 \text { cores } \\
\text { Lista de Adjetivos (Professor real e ideal) } \\
\text { Meus Talentos } \\
\text { Pensando a Criatividade com Palavras } \\
\text { Problemas Aritméticos } \\
\text { Problemas Verbais } \\
\text { Prova de Conservação de Comprimento de } \\
\text { Piaget }\end{array}$ & $\begin{array}{l}\text { Prova de leitura oral e escrita por ditado } \\
\text { Prova de memória de configuração } \\
\text { simples } \\
\text { Prova do Cheio e Vazio } \\
\text { Prova Piagetiana de Conservação de } \\
\text { Comprimento } \\
\text { Provas de Seriação Baseadas no Método Clínico } \\
\text { Piagetiano } \\
\text { Provas para avaliar o nível de variação } \\
\text { lingüística, leitura e escrita } \\
\text { Situações de tomada de atitudes } \\
\text { Tarefa de consciência fonológica e sintática } \\
\text { Tarefa de leitura e escrita de palavras reais e } \\
\text { inventadas } \\
\text { Tarefa de memória verbal } \\
\text { Textos estruturados com silogismos }\end{array}$ \\
\hline
\end{tabular}

analisados se concentraram acima do número máximo de doze vocábulos. Entende-se que esse limite possibilita espaço suficiente para se intitular um trabalho, visto que títulos muito pequenos, em alguns casos, podem revelar pouco esclarecimento para 0 leitor sobre o real conteúdo que será lido. Sugere-se que os autores sejam mais concisos e empreguem palavras adequadas que expressem a real natureza do trabalho, sem se tornar prolixo.

Outros itens analisados nos artigos foram a quantidade de participantes/sujeitos e sua respectiva escolaridade. No primeiro item chama atenção o fato de que 21 resumos apresentaram seus sujeitos de pesquisa de forma genérica, não podendo se ter uma idéia de características essenciais dos participantes. Entende-se que essa informação no resumo acadêmico, não pode ser ignorada, visto que muitas vezes os trabalhos nas bases de dados são rastreados justa- mente pela tipicidade da população pesquisada. $O$ resumo acadêmico não deve omitir esse dado, pois compromete a qualidade dele. Outro aspecto importante é que apenas 8 trabalhos utilizaram amostras maiores do que 1000 pessoas, talvez isso se deva principalmente da dificuldade de se trabalhar com amostras mais amplas.

No que concerne à etapa de escolarização dos participantes das pesquisas analisadas, novamente não houve distribuição eqüitativa. Duas etapas da educação formal predominaram, o ensino fundamental de $I^{\mathrm{a}}$ a $4^{\mathrm{a}}$ séries e o ensino superior. Esses resultados mostram que as séries iniciais são preferencialmente pesquisadas. Assim, hipotetiza-se que essas séries são a base da escolarização, inclusive na ascensão para as outras etapas da educação. No entanto, no caso do ensino fundamental supõe-se que os estudos realizados nessa fase sejam, em sua maioria, amostras compostas por con- 
veniência, considerando a maior facilidade de acesso em razão do interesse da escola em identificar o perfil de características psicológicas dos alunos.

A análise dos instrumentos empregados nas avaliações, conforme consta no Quadro I apontou que há uma predominância no uso de recursos psicométricos/fatoriais em comparação a outros meios de avaliação. Esse resultado expressa que, de um modo geral, os pesquisadores elegem técnicas que apresentam alguma fundamentação estatística para realizar sua avaliação, o que pode gerar resultados mais confiáveis. Ainda que se trabalhe com o erro de medida, tais técnicas são mais pontuais em detrimento de outros recursos que apresentam um maior grau de subjetividade. Poucos foram os estudos que trabalharam com esse recurso avaliativo.

Vale destacar que se optou por não trabalhar com a categorização dos instrumentos, o que geraria dados de freqüência e porcentagem, mas sim com a citação integral de todos os instrumentos empregados nas avaliações. O intuito dessa opção foi para que as portas não fossem fechadas, mas que houvesse $o$ interesse de outros pesquisadores para levantar novos questionamentos acerca dos atributos avaliados por meio desses instrumentos. Ao vislumbrar os vários instrumentos ali elencados, pode-se aventar que muitos aspectos importantes relacionados ao contexto escolar e educacional ainda não foram objetos de investigação psicológica, o que sugere a necessidade de que novos estudos sejam realizados nesse contexto.

\section{Considerações Finais}

Quando se propõe uma análise bibliométrica da produção científica em psicologia, diversos aspectos devem ser considerados. Dentre eles, destaca-se o acesso aos periódicos que foram selecionados. Por vezes as bibliotecas institucionais não apresentam a coleção completa dos periódicos, desse modo, as bases de dados são importantes recursos para implementar um trabalho de análise da produção científica.
Neste trabalho, em especial, a base de dados SciELO foi bastante utilizada, tendo em vista que parte dos periódicos analisados estavam indexados nessa base. Em todos os periódicos analisados observou-se um aumento da produção científica. Esse dado corrobora o fato de que a produção científica brasileira tem crescido e ganhado espaço no cenário científico nacional (Meneghini, 1998; Yamamoto e cols., 1999).

Trabalhos que focam a análise da produção científica deveriam ser freqüentes na área da psicologia. A meta-análise poderia funcionar como um crivo que indicaria a qualidade da produção científica já que nem todos os periódicos nacionais encontramse indexados no Institute for Scientific Informaticon (ISI) (Izique, 2002).

As pesquisas de Carelli (2002), Neves e cols. (2002), Santos e cols. (2003) e Bariani e cols. (2004) evidenciam que passos foram dados na realização de pesquisas que buscam avaliar a produção científica produzida na psicologia escolar e educacional. Sob esse aspecto, espera-se que esse estudo se configure no primeiro de uma série que fomentará novas investigações acerca da produção científica sobre o uso da avaliação psicológica nas várias etapas da educação formal.

\section{Referências}

American Psychologial Association (200I). Manual de publicações da American Psychological Association ( $4^{\mathrm{a}}$ ed.). Porto Alegre: Artmed.

Azevedo, J. M. L., \& Aguiar, M. A. (200I). A produção do conhecimento sobre a política educacional no Brasil: um olhar a partir da ANPED. Educação e Sociedade, 22(77), 49-70.

Bariani, I. C. D., Buin, E., Barros, R. C., \& Escher, C. A. (2004). Psicologia escolar educacional no ensino superior: análise da produção científica. Psicologia Escolar e Educacional, 8(I), 17-27.

Carelli, A. E. (2002). Produção científica em leitura: dissertações e teses (1990-1999). Tese de Doutorado, Centro de Ciências da vida, Pontifícia Universidade Católica de Campinas, Campinas. 
Coimbra Jr., C. E. A. (1999). Produção científica em saúde pública e as bases bibliográficas internacionais. Cadernos de Saúde Pública, 15(4), 883-888.

Costa, A. L. F. (2006). Publicações e avaliações de periódicos científicos: paradoxos de classificação QUALIS em psicologia. Dissertação de Mestrado, Programa de Pós-Graduação da Universidade Federal do Rio Grande do Norte, Rio Grande do Norte, Natal.

Figueira, I., Leta, L., \& De Meis, L. (1999). Avaliação da produção científica dos principais periódicos brasileiros de psiquiatria no período de 198I a 1995. Revista Brasileira de Psiquiatria, 2 I (4), 20I-208.

Freitas, M. H. A. (1998). Avaliação da produção científica: considerações sobre alguns critérios. Psicologia Escolar e Educacional, 2(3), 2 I I-228.

Izique, C. (2002). Produção crescente. Pesquisa FAPESP, (8I), 18-22.

Joly, M. C. R. A. (2000). A formação do Psicólogo escolar e a educação no terceiro milênio. Psicologia Escolar e Educacional, $4(2), 5 \mathrm{I}-55$.

Meneghini, R. (1998). Avaliação da produção científica e o projeto SciELO. Ciência da Informação [On-line], 27, (2), [citado em 28 de setembro de 2002]. Disponível: http:// www.scielo.br.

Meneghini, R., \& Fonseca, L. (1990). Índices alternativos de avaliação da produção científica em bioquímica no Brasil. Ciência e Cultura, 42, 629-645.

Mostafá, S. P., \& Máximo, L. F. (2003). A produção científica da Anped e da Intercom no GT da educação e comunicação. Ciência da Informação, 32(1), 96-101.

Neves, M. M. B. J., Almeida, S. F. C., Chaperman, M. C. L., \& Batista, B. P. (2002). Formação e atuação em psicologia escolar: análise das modalidades de comunicações nos congressos nacionais de psicologia escolar e educacional. Psicologia Ciência e Profissão, 22(2), 2-II.

Noronha, D. P. (1998). Análise das citações das dissertações de mestrado e teses de doutorado em saúde pública (19901994): estudo exploratório. Ciência da Informação [On-line], 27(I), [citado em 4 de outubro de 2002]. Disponível: http:// www.scielo.br

Noronha, A. P., Vendramini, C. M. M., \& Freitas, F. A. (2004). A avaliação psicológica no I Congresso Brasileiro de Psicologia. Em C. Machado, L. S. Almeida, M. Gonçalves \& V. Ramalho
(Orgs.), Avaliação Psicológica: formas e contextos (pp. II-I5). Braga: Psiquilíbrios Edições.

Oliveira, K. L., Cantalice, L., Joly, M. C. R. A., \& Santos, A. A. A. (2006). Produção científica de 10 anos da revista Psicologia Escolar e Educacional (1996/2005). Psicologia Escolar e Educacional, I0(2), 283-292.

Oliveira Filho, R. S., Hochman, B., Nahas, F. X., \& Ferreira, L. M. (2005). Fomento à publicação científica e proteção do conhecimento científico. Acta Cirúrgica Brasileira, 20 (2 suplemento), 35-39.

Sampaio, M. I. C., Sabadini, A. A. Z. P., \& Linguanotto, A. K. J. (2002). Periódicos Científicos: características e exigências. Mudanças, 10, I84-200.

Santos, A. A. A., Oliveira, K. L., \& Joly, M. C. A. (2003). Produção científica da área de escolar no I Congresso Brasileiro de Psicologia Ciência e Profissão. Psicologia Escolar e Educacional, 7(2), I35-| 44.

Sisto, F. F., Sbardelini, E. T. B., \& Primi, R. (200I). Contextos e questões da avaliação psicológica. São Paulo: Casa do Psicólogo.

Souza Filho, M. L., Belo, R., \& Gouveia, V. V. (2006). Testes Psicológicos: análise da produção científica no período de 2000-2004. Psicologia: Ciência e Profissão, 26(3), 478-489.

Witter, G. P. (1979). A pesquisa educacional nas primeiras Reuniões da SBPC. Em G. P. Witter (Org.), Pesquisas Educacionais (pp. 15-23). São Paulo: Símbolo.

Witter, C. (1996). Psicologia Escolar: produção científica, formação e atuação (1990-1994). Tese de doutorado, Universidade de São Paulo, São Paulo.

Witter, G. P. (1999). Metaciência e leitura. Em G. P.Witter (Org.), Leitura: textos e pesquisas (pp. 13-22). Campinas: Alínea.

Witter, G. P. (Org.). (2005). Metaciência e psicologia. Campinas: Alínea.

Witter, G. P. (2006). Envelhecimento: referenciais teóricos e pesquisas. Campinas: Alínea.

Yamamoto, O. H., Souza, C. S., \& Yamamoto, M. E. (1999). A produção científica na psicologia: uma análise dos periódicos brasileiros no período de 1990-1997. Psicologia: Reflexão e Crítica, I2(2), 549-565.

Zanella, A. V., \& Titon, A. P. (2005). Análise da produção científica sobre criatividade em programas brasileiros de pósgraduação em psicologia (1994-200 I). Psicologia em Estudo, I0(2), 305-316. 
Recebido em: 21/06/2007

Revisado em: 02/07/2007

Aprovado em: 28/0 I/2008

Sobre as autoras:

Katya Luciane de Oliveira (Katya.oliveira@saofrancisco.edu.br) - Psicóloga. Mestre em Psicologia pelo Programa de Pós-Graduação Stricto Sensu em Psicologia da Universidade São Francisco; Doutoranda em Psicologia, Desenvolvimento Humano e Educação - Faculdade de Educação - UNICAMP. Docente do Curso de Psicologia da Universidade São Francisco.

Acácia Aparecida Angeli dos Santos (acácia@saofrancisco.edu.br) - Psicóloga. Doutora em Psicologia Escolar e do Desenvolvimento Humano pela USP, docente da graduação no curso de psicologia e no Programa de Pós-graduação Stricto-sensu em Psicologia, da Universidade São Francisco-SP. Bolsista produtividade do CNPq.

Ana Paula Porto Noronha (ana.paula.noronha@terra.com.br) - Psicóloga. Doutora em Psicologia pela PUC-Campinas, docente da graduação no curso de psicologia e no Programa de Pós-graduação Stricto-sensu em Psicologia, da Universidade São Francisco-SP. Bolsista produtividade do CNPq.

Evely Boruchovitch (evely@unicamp.br) - Psicóloga. Ph.D em Educação pela University of Southern Califórnia, docente da graduação e da pósgraduação do Departamento de Psicologia Educacional da Faculdade de Educação da UNICAMP. Bolsista produtividade do CNPq.

Cláudia Araújo da Cunha (ccunha@uber.com.br) - Psicóloga. Mestre em Psicologia pela Universidade Gama Filho. Doutora em Educação - Faculdade de Educação da UNICAMP. Docente da Universidade Federal de Uberlândia.

Marucia Patta Bardagi (marucia.bardagi@gmail.com) - Psicóloga. Mestre e Doutora em Psicologia pela Universidade Federal do Rio Grande do Sul. Docente da Universidade Luterana do Brasil e Universidade Santa Cruz do Sul.

Simone F. da Silva Domingues (dominguessimone@zipmail.com.br) - Psicóloga. Mestre e Doutora em Educação pela PUC-SP. Docente da Universidade Cruzeiro do Sul. 